\title{
Cavitation bubble dynamics during pulsed laser ablation of a metallic glass in water
}

\author{
M.Q. Jiang ${ }^{\mathrm{a}, c, *}$, X.Q. Wu ${ }^{\mathrm{b}}$, Y.P. Wei ${ }^{\mathrm{b}}$, G. Wilde $^{\mathrm{d}}$, L.H. Dai ${ }^{\mathrm{a}, \mathrm{c}, *}$ \\ a State Key Laboratory of Nonlinear Mechanics, Institute of Mechanics, Chinese Academy of Sciences, Beijing 100190, China \\ ${ }^{\mathrm{b}}$ Key Laboratory of Mechanics in Fluid Solid Coupling Systems, Institute of Mechanics, Chinese Academy of Sciences, Beijing 100190, PR China \\ ${ }^{c}$ School of Engineering Science, University of Chinese Academy of Sciences, Beijing 100049, China \\ ${ }^{d}$ Institute of Materials Physics, Westfälische Wilhelms-Universität Münster, Münster 48149, Germany
}

\section{G R A P H I C A L A B S T R A C T}

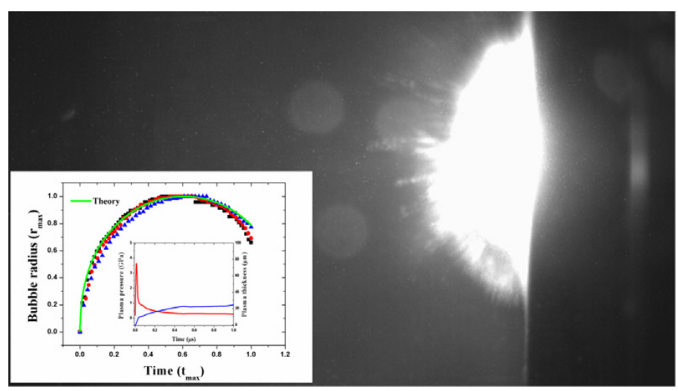

\section{A R T I C L E I N F O}

\section{Article history:}

Received 2 November 2016

Received in revised form

22 November 2016

Accepted 22 November 2016

Available online 1 December 2016

\section{Keywords:}

Metallic glasses

Pulsed laser ablation

Cavitation

Bubble dynamics

\begin{abstract}
A B S T R A C T
We report a cavitation bubble formation in water induced by nanosecond pulsed laser ablation of a $\mathrm{Zr}$ based (Vitreloy 1) bulk metallic glass target. Only the first bubble occurs due to an explosive-boiling-type ablation of the target. A theoretical model is developed to quantitatively describe the bubble nucleation and its initial growth. The results demonstrate that the laser-induced plasma can induce the nucleation of the bubble. Furthermore, it is revealed that the initial bubble growth is approximately adiabatic and inertial, obeying the Rayleigh-Plesset theory, albeit the significant ablation. This work sheds insight into the mechanics of water-confined laser ablation of metallic glasses, and provides guidance for synthesizing amorphous nanoparticles.
\end{abstract}

(C) 2016 Elsevier Ltd. All rights reserved.

\section{Introduction}

Cavitation bubbles are frequently observed during laser ablation of solids in liquids. This intriguing phenomenon has versa-

\footnotetext{
* Corresponding authors at: State Key Laboratory of Nonlinear Mechanics, Institute of Mechanics, Chinese Academy of Sciences, Beisihuanxi Road 15, Beijing 100190, China.

E-mail addresses: mqjiang@imech.ac.cn (M.Q. Jiang), lhdai@lnm.imech.ac.cn (L.H. Dai).
}

tile applications ranging from nanoparticle synthesis [1,2], surface cleaning [3] to luminescence [4]. The dynamics of cavitation bubble is of central importance since it reflects the fundamental response of liquid-plasma-solid system to laser ablation, and thus has drawn increasing interest [5-8]. Usually, the conventional Rayleigh-Plesset (R-P) theory is modified to reproduce the ablation-induced bubble dynamics. It is demonstrated that the initial evolution of a bubble is adiabatic and driven by inertial forces [7], but its long-term dynamics should take into account the target's ablation and the energy or mass exchange from and to the bubble [8]. In recent years, the pulsed-laser-ablation technology 
has been applied to a novel type of amorphous solids, i.e., metallic glasses, that are frozen from highly viscous melts via the glass transition $[9,10]$. This application has remarkably broadened the utilization of metallic glasses as advanced materials [11,12], and on the other hand, allows re-examining the traditional picture of laser-solid interaction [13-15]. It is noted that, however, laser ablation of metallic glasses was so far mostly performed in gaseous conditions, with only one exception [16]. Up to now, it still remains unexplored how a cavitation bubble emerges from a laser-ablated metallic glass target within a liquid environment.

In this letter, we experimentally observed the formation of cavitation bubbles during pulsed laser ablation of a typical $\mathrm{Zr}$ based bulk metallic glass within a water environment. A theoretical model is developed to quantitatively describe the initial bubble dynamics, where the bubble nucleation is mediated by the plasma evolution and its growth obeys the R-P theory.

\section{Material and methods}

A typical $\mathrm{Zr}_{41.2} \mathrm{Ti}_{13.8} \mathrm{Cu}_{12.5} \mathrm{Ni}_{10.0} \mathrm{Be}_{22.5}$ (at.\%, Vitreloy 1) bulk metallic glass was chosen as the target material. Targets $(10 \mathrm{~mm} \times$ $10 \mathrm{~mm} \times 2 \mathrm{~mm}$ ) were cut by wire electrical discharge machining of the as-cast material. Then, the target surfaces were polished to remove oxides. The glassy nature of the targets was confirmed by $\mathrm{X}$-ray diffraction and differential scanning calorimetry. The targets were submerged in filtered, de-ionized water. Single pulse ablation experiments were performed by irradiation with a Q-switched $\mathrm{Nd}$ : YAG laser operating at a wavelength of $1064 \mathrm{~nm}$ and a maximum output energy of $2.5 \mathrm{~J}$ per shot. The temporal profile of the laser pulse is in the near-Gaussian distribution with a full width of at half maximum (FWHM) of about 10 ns. The spatial profile of the laser pulse is modulated to nearly flat shape. The incident laser beam is focused with a quartz lens of nominal focal length of $600 \mathrm{~mm}$. The laser beam was normal to the target surface, and the beam path length in water is about $20 \mathrm{~mm}$, minimizing the possibility of nonlinear absorption of laser in water. The beam spot size is controlled to be about $2.7 \mathrm{~mm}$ in diameter. We repeated the experiments for three times. A new target was used for each experiment, but the water was not changed. A Photron Fastcam SA-X camera with a framing rate of 50,000 fps was used for the tracking of cavitation bubbles induced by the laser ablation. The morphologies of the irradiated areas were examined by using an FEI Sirion scanning electron microscope.

\section{Results and discussion}

Fig. 1 (and Supplementary Movie, Appendix A) shows the bubble evolution in water after the ablation of the Vitreloy 1 target. The first image captures the optically active plasma plume that has already expanded into the entire viewing window. Since the plasma lifetime is much shorter than the time between two frames [17-19], the first snapshot is regarded as a reference for time recording $(t=0 \mu \mathrm{s})$. The bubble expands for a duration of about $800 \mu \mathrm{s}$ and then shrinks, followed by the collapse after $1440 \mu \mathrm{s}$. The maximum bubble radius exceeds $8 \mathrm{~mm}$. It is noteworthy that inside the initially expanding bubble, and lasting for at least $100 \mu \mathrm{s}$, bright high-temperature matter is ejected from the target surface. This indicates that an explosive boiling process has taken place in the Vitreloy 1 glass after the water-confined ns-pulsed laser ablation [14]. The glassy target should have experienced an explosion-like vaporization from its superheated liquid, resulting in a violent ejection of a hightemperature mixture of vapor and droplets. We indeed observed that some ablated matter rapidly penetrated through the boundary of the bubble. Unfortunately, the present camera resolution cannot identify the size of these matter. With the unstable shrinking of the first bubble, the stored ablated matter finally destroys the bubble, leading to its collapse without rebounds (see the last six snapshots in Fig. 1)

We further examined the laser-irradiated area, as shown in the inset of Fig. 2(a). It is found that the actual irradiated area $S$ is very close to the focused beam spot size. Therefore, the maximum laser intensity $I_{\max }$ is estimated to be about $4 \mathrm{GW} / \mathrm{cm}^{2}$. This value is comparable to the explosive boiling thresholds for some crystalline and glassy metals [14,20,21]. On the edge of the irradiated area, surface ripples can be clearly observed (see Fig. 2(a)), implying that significant thermo-hydrodynamic instabilities occurred due to the present water-confined laser ablation [13,16]. Fig. 2(b) exhibits the typical ablation pattern. An enlarged view of the region " $C$ " in Fig. 2(b) is presented in Fig. 2(c). Fig. 2(d) corresponds to the region " $D$ " in Fig. 2(c). It can be seen that large numbers of molten-like droplets are distributed in the entire ablated area. These droplets are at a microscale and display an actinomorphic distribution around certain locally ablated sites (see Fig. 2(d)). This ablation pattern is very different from that observed after ablation in air where the thermal ablation dominates [14]. In the present ablation in aqueous environment the confinement of cavitation bubbles induces a more severe explosive boiling of higher-degree superheated target material, leading to a coupled thermo-mechanical ablation. This is consistent with the violent ejection of bright ablated matter observed in Fig. 1. However, we find that there is no ablated matter deposited back on the ablated area after the bubble collapsed. A possible reason is that, for a longlived bubble, the delayed shrinking and collapse guarantees that a substantial amount of the ablated matter stored in the bubble can be delivered into the water instead of being deposited back on the target surface. Nevertheless, some nanoparticles with an average size of about $50 \mathrm{~nm}$ can be observed at the edge of the irradiated area (see Fig. 2(e) and (f)). We believe that these deposited nanoparticles belong to the ablated matter that penetrates through the boundary of the bubble. These results support the idea that the bubble cavity is an important site for nanoparticle generation [1,2].

Since the ablated matter mainly enters the water, the laser pulse energy for subsequent pulses might be reduced. This could affect the bubble dynamics in latter ablations, if the water is not changed. Fig. 3(a) shows the temporal evolution of the bubble radius, respectively, corresponding to three sequential ablations. Obviously, the decrease of the pulse energy decreases the maximum radius $\left(r_{\max }\right)$ and lifetime $\left(t_{\max }\right)$ of the bubbles. Nevertheless, if the bubble radius and time are normalized by $r_{\max }$ and $t_{\mathrm{max}}$, respectively, the three curves of the bubble dynamics will collapse into one (see Fig. 3(b)). This implies that the underlying mechanism for the dynamics of these bubbles is identical at least in the early stage, although their absolute values may be different.

It is well known that the bubble dynamics, i.e., the temporal variation of bubble radius $R(t)$, can be described by the RP equation

$\rho_{W}\left(R \ddot{R}+\frac{3}{2} \dot{R}^{2}\right)=P_{B}(t)-P_{\infty}-2 \frac{\sigma}{R}-4 \frac{\eta \dot{R}}{R}$,

where $P_{B}(t), \rho_{W}, P_{\infty}, \sigma$ and $\eta$, respectively, denote the internal pressure of the bubble, the mass density, the far-field pressure, the surface tension and the dynamic viscosity of the surrounding liquid. For the water-confined ns-pulsed laser ablation of metals, the surface tension and viscosity effects can be neglected if the bubble size and lifetime are of the order of $10^{-3} \mathrm{~m}$ and $10^{2} \mu \mathrm{s}$, respectively [7]. However, the precise solution of the simplified $\mathrm{RP}$ equation is still challenging. It usually requires the nucleation state of a bubble as additional adjusting parameters [8] or resorts to the experimental measurements $[7,22]$. Actually, the bubble nucleation originates from the fast evolution of laser-induced plasma, which has been confirmed experimentally [18,23]. 


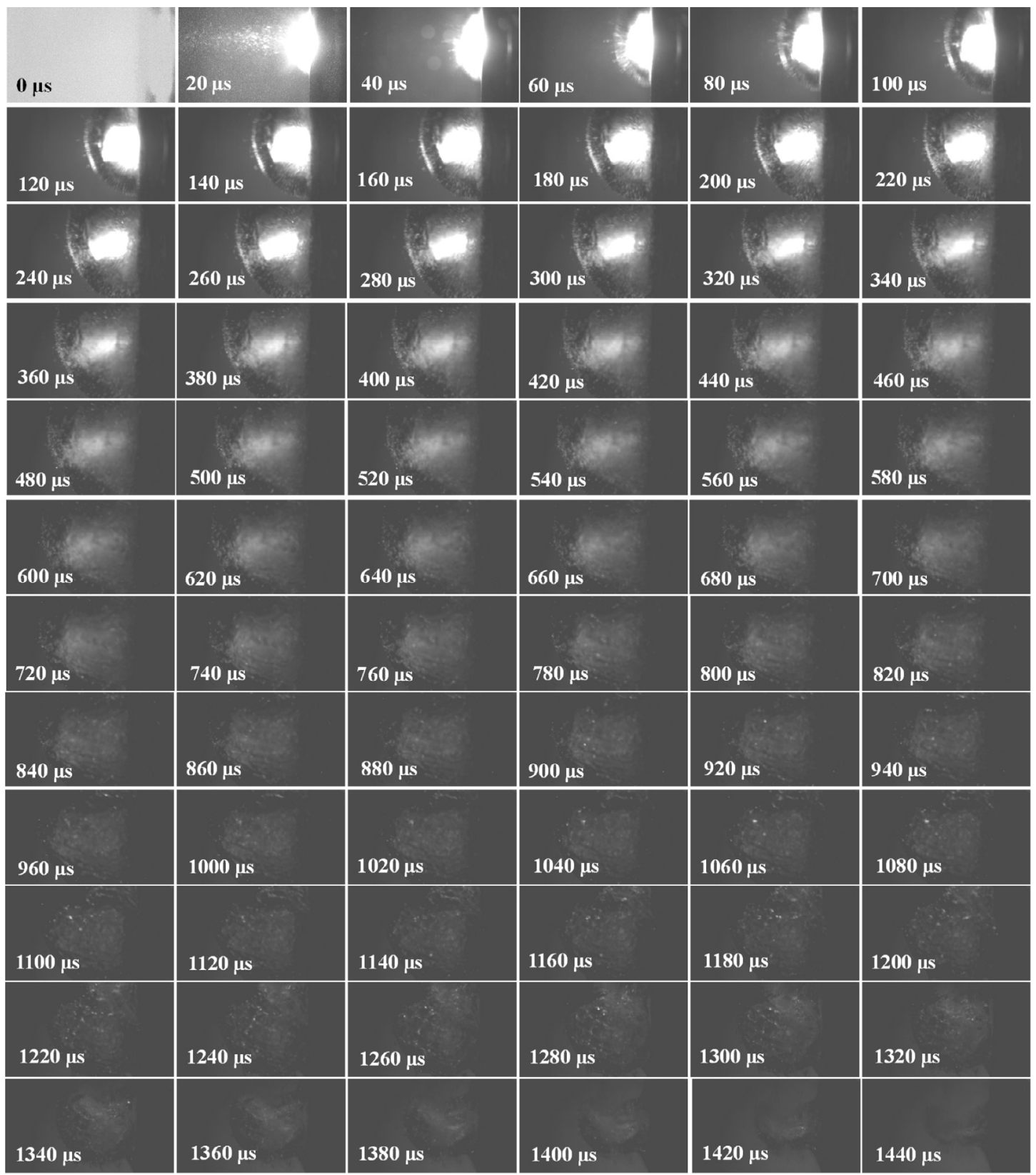

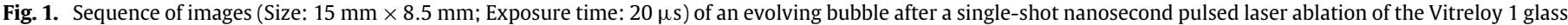
target in water.

When the laser pulse with a near-Gaussian intensity profile $I(t)$ is focused on the Vitreloy 1 target through water, atomization and ionization of the irradiated spot occur and a plasma is thus produced. It is reasonable to assume that the incident laser energy is mainly absorbed by the plasma, which is transferred to the internal energy of the plasma and the external work against the plasma boundaries [24-26]

$S I(t) \mathrm{d} t=\mathrm{d} W+\mathrm{d} e$,

where $\mathrm{d} W=p(t) \mathrm{d} H(t)$ with the average pressure $p(t)$ and the thickness $H(t)$ of the plasma. Following Fabbro et al., [26] a constant fraction $\alpha$ of the internal energy of the plasma represents the thermal energy $e_{T}$, and the fraction $(1-\alpha)$ being the ionization energy. Regarding the plasma as an ideal gas [24-26], $e_{T}$ can be expressed by $e_{T}=3 p(t) H(t) / 2$. Eq. (2) then becomes

$S I(t)=p(t) \frac{\mathrm{d} H(t)}{\mathrm{d} t}+\frac{3}{2 \alpha} \frac{\mathrm{d}}{\mathrm{d} t}[p(t) H(t)]$.
After the laser pulse is switched off at $t>\tau$ (pulse width $10 \mathrm{~ns}$ ), the plasma expands adiabatically,

$p(t)=p(\tau)\left[\frac{H(\tau)}{H(t)}\right]^{\gamma}$

where $\gamma$ is the adiabatic exponent of the plasma. The initial conditions are:

$H(0)=0$ and $p(0)=0$.

The boundary conditions are:

$\mathrm{d} H(t) / \mathrm{d} t=u_{W}(t)+u_{T}(t)$,

where $u_{W}(t)$ and $u_{T}(t)$ are the moving velocities at the interfaces between the plasma layer and its neighbors, i.e., the confining water and the Vitreloy 1 target, respectively. The two terms are determined by the dynamic response of the water-target system 

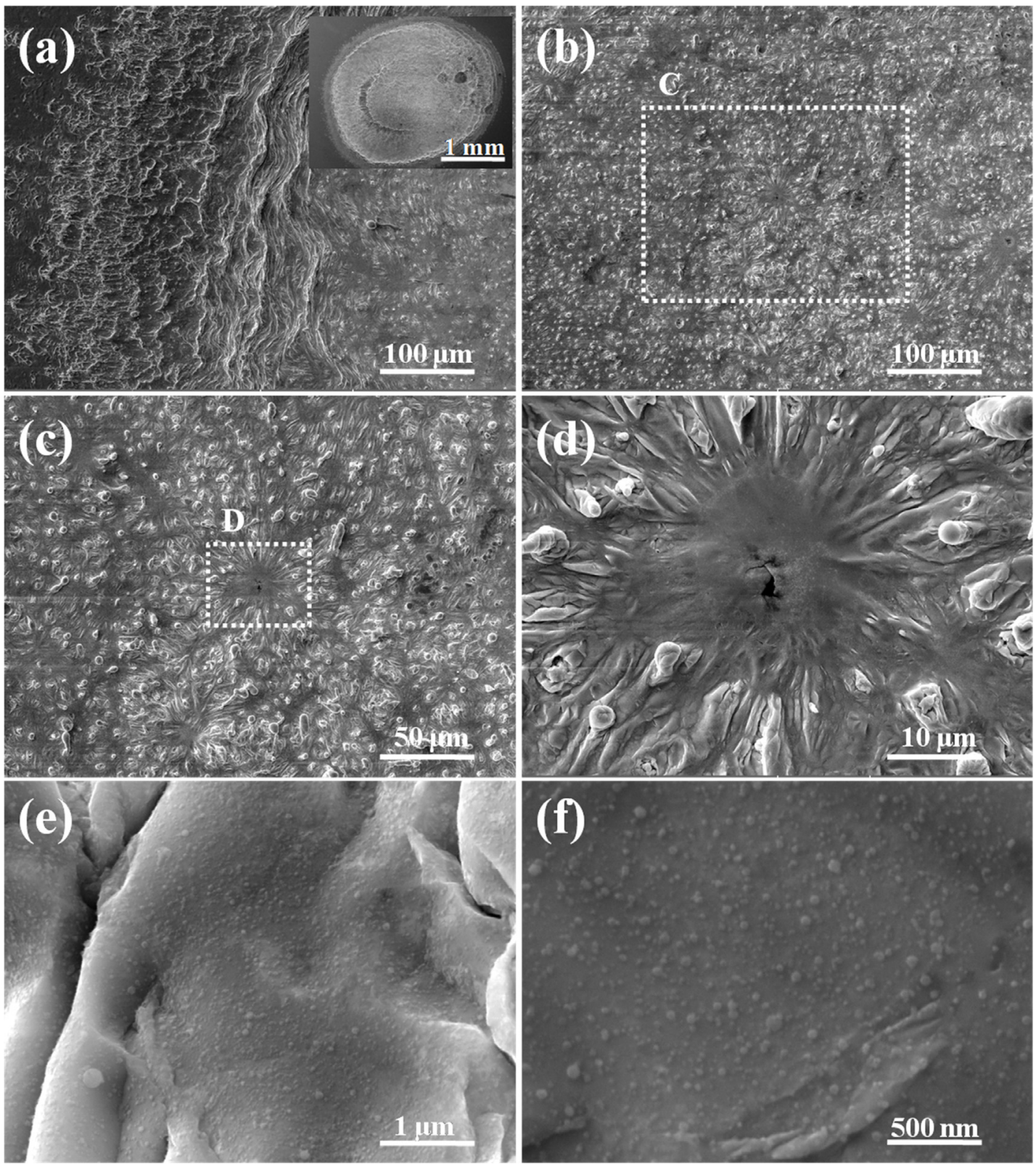

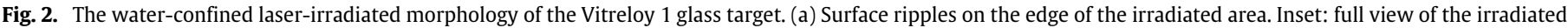
area. (b)-(d) Typical ablation patterns at different magnifications. (e) and (f) Nanoparticles deposited back on the edge of the irradiated area.

under the plasma pressure $p(t)$ and its time $\dot{p}(t)$, i.e. [25],

$u_{W}(t)=\frac{p(t)}{\rho_{W} c_{W}} ; \quad u_{T}(t)=\frac{p(t)}{\rho_{T} c_{T}}, \quad t \leq \tau$.
$u_{W}(t)=f(p(t), \dot{p}(t)) ; \quad u_{T}(t)=g(p(t), \dot{p}(t)), \quad t>\tau$

where $c_{W}$ and $c_{T}$ are the longitudinal sound velocities in water and in the target, respectively. The functions $f$ and $g$ are determined by the equation of state of the water [27] and the elastic-perfectly plastic constitution of the Vitreloy 1 target [28]. In order to obtain the plasma evolution, $p(t)$ and $H(t)$, Eqs. (3)-(8) should be solved simultaneously. To this end, a coupled explicit finite differential code with first order precision is developed [24,25], by combining the LS-DYNA explicit package [29] with the LS-PREPOSTD code. The relevant properties/parameters of the calculations are given in Supplementary Table 1 (Appendix A).

The calculated temporal variation of the plasma pressure $p(t)$ and the thickness $H(t)$ is shown in the inset of Fig. 3(b). Within the pulse width, the plasma pressure increases rapidly and reaches a peak of about 3.79 GPa at $12.5 \mathrm{~ns}$. After extinguishing of the laser pulse, the plasma cools down and its pressure rapidly attenuates to a relatively stable level of $10^{2} \mathrm{MPa}$ after about $500 \mathrm{~ns}$. At that moment, the plasma expansion, which firstly experienced a fast-growing stage, also becomes stable. These calculated results are consistent with the observation that the plasma reaches the maximum volume after a few hundred nanoseconds [18,23]. Wu et al. [19] have also found that, for the $\sim 1 \mathrm{GW} / \mathrm{cm}^{2}$ laser ablation, the plasma length can only expand in $1 \mu \mathrm{s}$ after the laser peak. Therefore, we reasonably choose the plasma state at $500 \mathrm{~ns}$ as the onset/nucleation point of the cavitation bubble. We then obtain the initial pressure $P_{B}(0) \approx 0.16 \mathrm{GPa}$ of the bubble. Based on the volume conservation, the initial radius $R_{0}$ of the bubble can be calculated by $4 \pi R_{0}^{3} / 3=H_{0} S$, where $H_{0} \approx 22.4 \mu \mathrm{m}$. For the first bubble, its initial evolution is usually assumed to be adiabatic [7]. In addition, we neglect mass transfer from and to the bubble, although it may affect the long-term dynamics of the bubble, as 

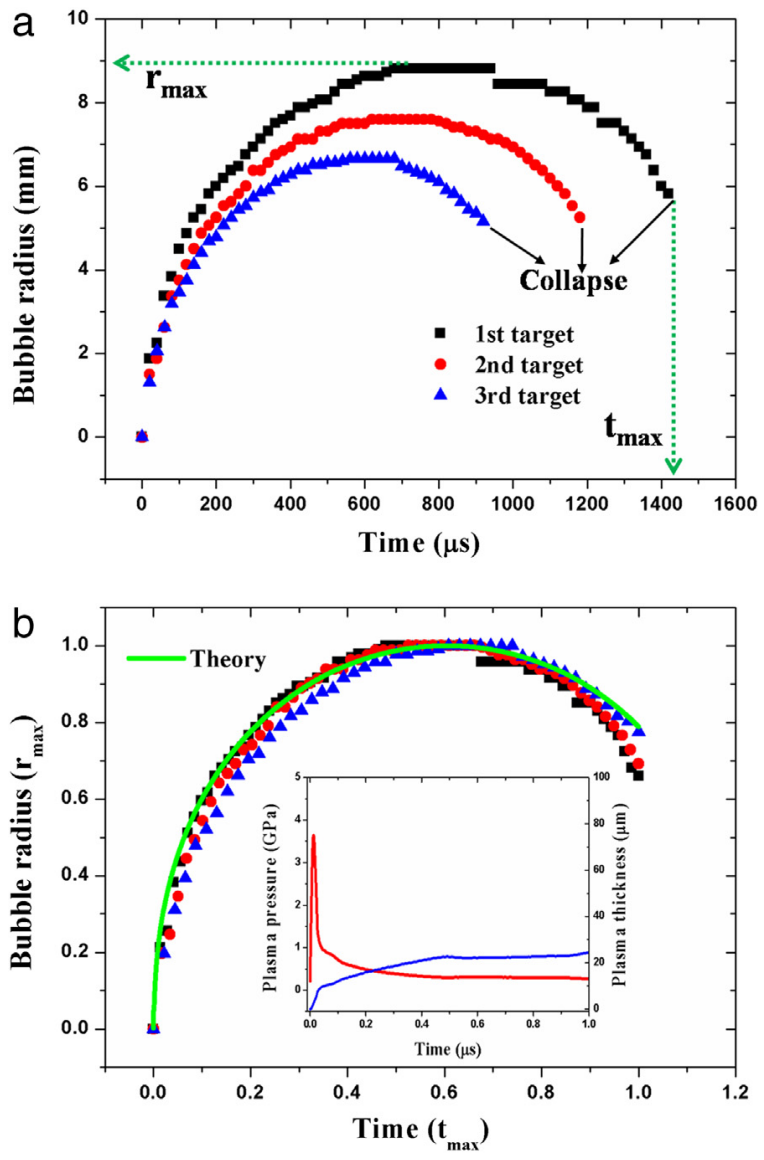

Fig. 3. (a) Temporal evolution of the bubble radius obtained after three sequential laser ablations of the Vitreloy 1 glass target in water. (b) Comparison between the experimental and theoretical results concerning the normalized bubble dynamics. Inset: the calculated time evolution of the plasma pressure (red curve) and thickness (blue curve). (For interpretation of the references to color in this figure legend, the reader is referred to the web version of this article.)

observed in Fig. 1. The bubble pressure thus follows

$P_{B}(t)=P_{B}(0)\left(\frac{R_{0}}{R(t)}\right)^{3 \varphi}$,

where $\varphi$ is the adiabatic exponent of the vapor. Uniting Eqs. (1) and (9), we theoretically reproduce the temporal variation of the bubble radius $R(t)$ after the ns-pulse laser ablation of the Vitreloy 1 target in water. After normalization, the calculated result is marked by a solid line in Fig. 3(b), which agrees well with the experimental measurements. Such agreement provides important clues for the dynamics of a cavitation bubble in water due to the ns-pulsed laser ablation of the Vitreloy 1 glassy target. First, the stable state of the plasma induced by laser ablation can be regarded as the nucleation point of the cavitation bubble. This process is theoretically modeled in the present letter, although its experimental evidence is clear $[18,23]$. Second, the initial evolution of the first bubble is accompanied by the target's ablation in the form of an explosive boiling [14]. But the latter does not significantly affect the former. In our model, therefore, mass exchange between the bubble and its environment is neglected. Nevertheless, the significant target's ablation should be responsible for the disappearance of the bubble rebound. Third, the bubble follows an approximately adiabatic evolution, where no thermal energy is transferred from and to the bubble, as recently revealed by Lam et al. [7]. At last, the bubble evolution is driven mainly by inertial forces. Both the surface tension and the viscosity effects can be neglected in the present case.

\section{Conclusions}

Single pulsed ablations of a Vitreloy 1 bulk metallic glass target were performed in water by a nanosecond laser. We observed the ablation-induced cavitation bubbles that can survive for more than $1000 \mu \mathrm{s}$ with a maximum radius of about $8 \mathrm{~mm}$. We propose a theoretical model to describe the initial bubble dynamics. This model considers the laser-induced plasma to initiate the nucleation of the bubble, and further assumes that the initial growth of the bubble is approximately adiabatic and driven by inertial forces. The calculated bubble dynamics is in agreement with that experimental results. Thus this work can yield deeper insight into the mechanics of water-confined laser ablation of metallic glasses and even provides a possible way to produce amorphous nanoparticles by laser ablation.

\section{Acknowledgments}

Financial support is from the National Nature Science Foundation of China (Grant Nos. 11522221, 11372315 and 11472287) and the Strategic Priority Research Program of the Chinese Academy of Sciences (Grant No. XDB22040302). GW acknowledges support by Deutsche Forschungsgemeinschaft.

\section{Appendix A. Supplementary data}

Supplementary material related to this article can be found online at http://dx.doi.org/10.1016/j.eml.2016.11.014.

\section{References}

[1] V.Amendola, M. Meneghetti, What controls the composition and the structure of nanomaterials generated by laser ablation in liquid solution? Phys. Chem. Chem. Phys. 15 (2013) 3027-3046

[2] S. Horoz, L. Lu, Q. Dai, J. Chen, B. Yakami, J.M. Pikal, W. Wang, J. Tang, CdSe quantum dots synthesized by laser ablation in water and their photovoltaic applications, Appl. Phys. Lett. 101 (2012) 223902.

[3] C.-D. Ohl, M. Arora, R. Dijkink, V. Janve, D. Lohse, Surface cleaning from laserinduced cavitation bubbles, Appl. Phys. Lett. 89 (2006) 074102.

[4] V. Lazic, S. Jovicevic, M. Carpanese, Laser induced bubbles inside liquids: Transient optical properties and effects on a beam propagation, Appl. Phys. Lett. 101 (2012) 054101.

[5] I. Akhatov, O. Lindau, A. Topolnikov, R. Mettin, N. Vakhitova, W. Lauterborn, Collapse and rebound of a laser-induced cavitation bubble, Phys. Fluids 13 (2001) 2805-2819.

[6] V. Kotaidis, A. Plech, Cavitation dynamics on the nanoscale, Appl. Phys. Lett. 87 (2005) 213102.

[7] J. Lam, J. Lombard, C. Dujardin, G. Ledoux, S. Merabia, D. Amans, Dynamical study of bubble expansion following laser ablation in liquids, Appl. Phys. Lett. 108 (2016) 074104

[8] W. Soliman, T. Nakano, N. Takada, K. Sasaki, Modification of Rayleigh-Plesset theory for reproducing dynamics of cavitation bubbles in liquid-phase laser ablation, Japan. J. Appl. Phys. 49 (2010) 116202.

[9] A.L. Greer, Metallic glasses, Science 267 (1995) 1947-1953.

[10] W.L. Johnson, Bulk glass-forming metallic alloys: science and technology, MRS Bull. 24 (1999) 42-56.

[11] M. Sorescu, Direct evidence of laser-induced magnetic domain structures in metallic glasses, Phys. Rev. B 61 (2000) 14338-14341.

[12] H. Huang, N. Jun, M.Q. Jiang, M. Ryoko, J. Yan, Nanosecond pulsed laser irradiation induced hierarchical micro/nanostructures on $\mathrm{Zr}$-based metallic glass substrate, Mater. Des. 109 (2016) 153-161.

[13] Y. Liu, M.Q. Jiang, G.W. Yang, Y.J. Guan, L.H. Dai, Surface rippling on bulk metallic glass under nanosecond pulse laser ablation, Appl. Phys. Lett. 99 (2011) 191902.

[14] M.Q. Jiang, Y.P. Wei, G. Wilde, L.H. Dai, Explosive boiling of a metallic glass superheated by nanosecond pulse laser ablation, Appl. Phys. Lett. 106 (2015) 021904.

[15] E. Williams, E.B. Brousseau, Nanosecond laser processing of Zr41.2Ti13.8Cu12.5Ni10Be22.5 with single pulses, J. Mater. Process. Technol. 232 (2016) 34-42.

[16] Y. Liu, M.O. Jiang, G.W. Yang, J.H. Chen, Y.J. Guan, L.H. Dai, Saffman-Taylor fingering in nanosecond pulse laser ablating bulk metallic glass in water Intermetallics 31 (2012) 325-329.

[17] X. Zeng, X.L. Mao, R. Greif, R.E. Russo, Experimental investigation of ablation efficiency and plasma expansion during femtosecond and nanosecond laser ablation of silicon, Appl. Phys. A 80 (2005) 237-241. 
[18] A. De Giacomo, M. Dell’Aglio, A. Santagata, R. Gaudiuso, O. De Pascale, P. Wagener, G.C. Messina, G. Compagnini, S. Barcikowski, Cavitation dynamics of laser ablation of bulk and wire-shaped metals in water during nanoparticles production, Phys. Chem. Chem. Phys. 15 (2013) 3083-3092.

[19] J. Wu, W. Wei, X. Li, S. Jia, A. Qiu, Infrared nanosecond laser-metal ablation in atmosphere: Initial plasma during laser pulse and further expansion, Appl Phys. Lett. 102 (2013) 164104.

[20] N.M. Bulgakova, A.V. Bulgakov, Pulsed laser ablation of solids: transition from normal vaporization to phase explosion, Appl. Phys. A 73 (2001) 199-208.

[21] W.T. Nichols, T. Sasaki, N. Koshizaki, Laser ablation of a platinum target in water. I. Ablation mechanisms, J. Appl. Phys. 100 (2006) 114911.

[22] W. Hentschel, W. Lauterborn, Acoustic emission of single laser-produced cavitation bubbles and their dynamics, Appl. Sci. Res. 38 (1982) 225-230.

[23] A. Tamura, A. Matsumoto, K. Fukami, N. Nishi, T. Sakka, Simultaneous observation of nascent plasma and bubble induced by laser ablation in water with various pulse durations, J. Appl. Phys. 117 (2015) 173304.
[24] X.Q. Wu, Z.P. Duan, H.W. Song, Y.P. Wei, X. Wang, C.G. Huang, Shock pressure induced by glass-confined laser shock peening: Experiments, modeling and simulation, J. Appl. Phys. 110 (2011) 053112.

[25] X.Q. Wu, Q.M. Tan, C.G. Huang, Geometrical scaling law for laser shock processing, J. Appl. Phys. 114 (2013) 043105.

[26] R. Fabbro, J. Fournier, P. Ballard, D. Devaux, J. Virmont, Physical study of laserproduced plasma in confined geometry, J. Appl. Phys. 68 (1990) 775-784.

[27] M.A. Meyers, Dynamic Behavior of Materials, Wiley-Interscience, New York, 1994.

[28] L.F. Liu, L.H. Dai, Y.L. Bai, B.C. Wei, J. Eckert, Behavior of multiple shear bands in Zr-based bulk metallic glass, Mater. Chem. Phys. 93 (2005) 174-177.

[29] J.O. Hallquist, LS-DYNA3D theoretical manual Livermore Software Technology Corporation, 1993. 